\title{
Correction to: Randomized community trial on nosocomial infection control educational module for nurses in public hospitals in Yemen: a study protocol
}

Gamil Alrubaiee ${ }^{1}$, Anisah Baharom², Ibrahim Faisal ${ }^{2}$, Kadir Shahar Hayati ${ }^{2}$, Shaffe Mohd. Daud ${ }^{3}$ and Huda Omer Basaleem ${ }^{4}$

\section{Correction to: BMC Nursing}

$$
\text { https://doi.org/10.1186/s12912-019-0333-3 }
$$

In the original publication of this article [1], a formula's format in the section of "Sample size calculation" was wrong and needs to be revised.

The correct formula should be:

$$
\mathbf{N}=\left[\mathbf{Z}_{(1-\alpha)} \sqrt{\mathbf{2 P}(\mathbf{1}-\mathbf{P})}+\mathbf{Z}_{(\mathbf{1}-\boldsymbol{\beta})} \sqrt{\mathbf{P 1}(\mathbf{1}-\mathbf{P 1})+\mathbf{P 2}(\mathbf{1}-\mathbf{P 2})}\right]^{2} /(\mathbf{P 1}-\mathbf{P 2})^{2} .
$$

The original article has been corrected.

\begin{abstract}
Author details
${ }^{1}$ Department of Applied Medical Sciences, Faculty of Medical Sciences,

Al-Razi University, Sana'a, Yemen. ${ }^{2}$ Department of Community Health, Faculty of Medicine and Health Sciences, Universiti Putra Malaysia, Seri Kembangan, Malaysia. ${ }^{3}$ Department of Foundations of Education, Faculty of Educational Studies, Universiti Putra Malaysia, Seri Kembangan, Malaysia. ${ }^{4}$ Department of Community Medicine and Public Health, Faculty of Medicine and Health Sciences, University of Aden, Aden, Yemen.
\end{abstract}

Published online: 18 April 2019

\section{Reference}

1. Alrubaiee, et al. BMC Nurs. 2019;18:10 https://doi.org/10.1186/s12912-019-
0333-3.

\footnotetext{
* Correspondence: anisbaharom@yahoo.com

${ }^{2}$ Department of Community Health, Faculty of Medicine and Health Sciences,

Universiti Putra Malaysia, Seri Kembangan, Malaysia
} 